\title{
Caracterización clínica y epidemiológica de pacientes con traumatismo raquimedular
}

\section{Clinical and epidemiological characterization of patients with spinal cord trauma}

\author{
Alvaro Alejandro García Villafuerte \\ Universidad de San Carlos de Guatemala \\ alvarogarcia0118@hotmail.com \\ https://orcid.org/0000-0001-5677-4521
}

Recibido: $14 / 07 / 2021$

Aceptado: 26/08/2021

\section{Referencia del artículo}

García Villafuerte, A. A. (2021). Caracterización clínica y epidemiológica de pacientes con traumatismo raquimedular. Revista Diversidad Científica, 1(1). 151-158.

DOI: https://doi.org/10.36314/diversidad.v1i1.16

\section{Resumen}

Para la identificación de las características clínicas y epidemiológicas de los pacientes con traumatismo raquimedular se buscaron aspectos de los pacientes que mayormente se ven involucrados. Se recopiló información sobre su nivel anatómico más frecuente, los signos clínicos y sus complicaciones secundarias. Toda esta amalgama de información se presenta para conocer el impacto de esta patología en un individuo y en la sociedad. OBJETIVO: identificar las características clínicas y epidemiológicas de pacientes con traumatismo raquimedular MÉTODO: se realizó una revisión basada en metaanálisis, artículos médicos y estudios previos. RESULTADOS: el género masculino y el grupo etario de 30 a 45 años sufren frecuentemente traumatismo medular asociado a accidentes automovilísticos y caídas. La región cervical es la más afectada. Los signos clínicos mayormente reportados son la paresia, alteraciones en la sensibilidad, hipotensión y las complicaciones secundarias al trauma raquimedular son insuficiencia respiratoria y shock medular. CONCLUSIÓN: el género masculino con mayor frecuencia presenta lesión medular principalmente en una edad productiva para la sociedad, donde la etiología involucra accidentes y caídas. La región cervical es más propensa a sufrir lesiones, ocasionando paresia, alteraciones en la sensibilidad, hipotensión, incontinencia urinaria. Si la lesión se agrava puede ocasionar insuficiencia respiratoria, shock medular e incluso la muerte.

Palabras clave: caracterización, traumatismo, medular 


\begin{abstract}
The characterization of the patient with spinal cord injury was based on the search for specific features that make up this pathology. Aspects such as gender, age and injury mechanism were searched to identify the patients who are mostly involved. Information was collected on the pathophysiology of the trauma, as well as on its anatomical level in which it was more frequent, the clinical signs presented by these patients and their secondary complications that aggravated the initial symptoms. All this amalgam of information is presented to know the impact of this pathology on an individual and on society.
\end{abstract}

Keywords: characterization, trauma, medullary 


\section{Introducción}

El traumatismo raquimedular las lesiones traumáticas que involucran conjunta o aisladamente los elementos que conforman la columna vertebral, desde uniones osteoligamentosas, músculos, vasos sanguíneos, hasta la médula espinal en cualquiera de sus niveles. La lesión medular ocasionada por el traumatismo conlleva un daño neurológico en el cual se produce el déficit de las funciones sensoriales y motoras, esto secundario a la daño total o parcial de la médula. (Padilla et al., 2017).

La patología traumática medular ha figurado dentro de los principales problemas de salud pública debido a que su intervención debe ser rápida, realizada por especialistas con experiencia, su recuperación es a largo plazo, implica altos costos para el sistema de salud y repercute en el paciente y su núcleo familiar. (Teherán et al., 2016).

Los traumatismos a nivel mundial se han convertido en una epidemia desatendida en los países en vías de desarrollo, pues estos llegan a ocasionar más de cinco millones de muertes al año. El estudio sobre la Carga de morbilidad mundial y factores de riesgo publicado en 1990 calculó que los traumatismos constituían en esa época más del $15 \%$ de la problemática en salud del mundo, las proyecciones estimadas para el año 2020 marcaban que esta cifra aumentaría al 20\%. (Teherán et al., 2016).

Las lesiones raquimedulares se ven acompañadas de politraumatismos en un $70 \%$ de los casos. La Organización Mundial de la Salud estima que cerca de medio millón de personas sufren lesiones medulares al año, en su mayoría hombres, de estas personas existe la probabilidad entre dos y tres veces mayor de presentar una muerte prematura principalmente en los países que presentan una tasa baja e intermedia de ingresos económicos. (Arriagada y Macchiavello, 2020)

En Guatemala, los hospitales de la red nacional especialmente en la región oriente, el Hospital Nacional de Chiquimula y el Hospital Regional de Zacapa día con día atienden a pacientes politraumatizados por accidentes automovilísticos, caídas, lesiones deportivas o hechos de violencia. Dado que la severidad del trauma raquimedular es potencialmente devastadora y sus lesiones se pueden prevenir, resulta necesario conocer su impacto, distribución en la población y comprender el efecto de la concientización a las personas sobre el cuidado de la columna vertebral para disminuir la gravedad de las lesiones. Así mismo, mejorar el acceso y la calidad de la atención médica, el proceso de rehabilitación oportuna de estos pacientes para mejorar su supervivencia, reducir la morbilidad, discapacidad física y dependencia. 
Los objetivos planteados para este ensayo consisten en explicar el perfil epidemiológico de pacientes con traumatismo raquimedular en base a género, edad y mecanismo de lesión. Describir los signos clínicos más frecuentes en traumatismo raquimedular. Identificar el nivel de la lesión medular más frecuente. Especificar las lesiones secundarias más frecuentes en pacientes con traumatismo raquimedular.

\section{Contenido}

El traumatismo raquimedular es una lesión de carácter mixto en donde el contenido neurológico y el componente óseo de la columna presentan alteraciones en su estructura y su función, lo cual conlleva a que la vía sensitiva, motora y autonómica que regula las actividades del cuerpo humano se encuentren abolidas. Los politraumatismos están fuertemente vinculados con la lesión medular, así como también los traumatismos craneoencefálicos. El diagnóstico certero en la etapa aguda de la lesión es vital para mejorar el pronóstico del paciente. (Bustamante, 2021).

La incidencia global es variable, pero se tiene un estimado de 23 casos por millón de personas. Al hacer una comparación entre regiones esta cifra puede cambiar, tal como muestra Norteamérica con 40 casos/millón, Europa reporta 15 casos/ millón, Australia 16 casos/millón. Actualmente no se cuenta con una incidencia establecida para nuestra región. Los varones de edades comprendidas entre los 25 y los 35 años se ven más afectado, mostrando una relación 4:1 con respecto a las mujeres. La mortalidad registrada en el lugar del incidente o en las salas de emergencia de los hospitales aumenta sus cifras hasta $79 \%$. Las áreas anatómicas más móviles de la columna vertebral se encuentran asociadas a las lesiones medulares, de estas la región cervical se ve comprometida hasta en un $75 \%$ de los traumatismos, específicamente entre C3-C7. La unión toracolumbar, a nivel de L1, se ve comprometida en el 16\% de los traumatismos. (Teherán et al., 2016).

Las lesiones inestables de la columna cervical son perjudiciales y muestran mayor susceptibilidad de desarrollar una lesión secundaria por una manipulación incorrecta del paciente. Los traumatismos contusos producen lesiones cervicales en el $5 \%$ de los pacientes, además puede generar lesiones inestables en el $14 \%$ de los casos. Lo cual explica que las tetraplejías y paraplejías se presenten con mayor frecuencia. Por el contrario, las lesiones ocasionadas a nivel torácico y lumbar son relativamente estables (Bustamante, 2021).

La etiología de esta lesión frecuentemente se ve asociada a los accidentes automovilísticos $(48 \%)$, las caídas de altura (16\%), hechos de violencia $(12 \%)$ y en 
algunas regiones los accidentes deportivos (10\%). Las principales causas pueden diferir en países en vías de desarrollo, en donde los hechos de violencia producidos por heridas por armas de fuego y arma blanca aumentan su incidencia. El nivel neurológico en el cual se reporta la mayor frecuencia de lesiones es el cervical en un $75 \%$ de los casos, seguido del nivel torácico con un $36 \%$ y el nivel lumbar con $17 \%$ de los casos. (Bustamante, 2021).

La fisiopatología del trauma medular presenta una lesión primaria y secundaria. La lesión primaria se desarrolla por la energía directa producida por el impacto del trauma inicial lo que ocasiona el desplazamiento de las estructuras de la columna vertebral. La lesión secundaria inicia generando cambios progresivos vasculares secundarios al trauma primario generando edema, isquemia, aumento en la producción de radicales libres, desequilibrio iónico, alteración en el metabolismo del calcio, llegando a desarrollar excitotoxicidad (Baabor et al., 2016).

La lesión medular secundaria inicia inmediatamente después del trauma inicial y puede permanecer por varias semanas. Se presenta un incremento progresivo del daño tisular, afectando la médula espinal a sus distintos niveles. además, el edema intracelular y la mayor permeabilidad generada por el daño endotelial es un factor clave en la extravasación de los componentes del sistema inmunitario. El trauma origina un componente de procesos fisiopatológicos los cuales se dividen en fases para una adecuada comprensión (Padilla et al., 2017).

La primera fase, denominada fase inmediata se presenta entre las 0 y 2 horas de ocurrido el traumatismo. La hemorragia de la sustancia gris y la inflamación son los componentes que se originan. La segunda, la fase aguda, comprendida entre las 2 y 48 horas del trauma, en la cual se produce trastorno en el metabolismo iónico, liberación de radicales libres, excitotoxicidad, secundario a la respuesta celular y sus mediadores inflamatorios. La tercera fase, la fase subaguda, ocurre entre 2 días y 2 semanas, inicia la respuesta fagocítica la cual es desencadenada por la respuesta inflamatoria, además se forma la cicatriz glial seguido de la restauración iónica. La cuarta fase, la fase intermedia, va desde 2 semanas hasta los 6 meses, en la cual culmina la cicatrización glial y la regeneración axonal y la fase final, denominada fase crónica, comprende desde los 6 meses en adelante, se produce maduración de la lesión con la consiguiente degeneración Walleriana (Baabor et al., 2016)

En el abordaje del paciente es recomendable la evaluación del "ABCD", priorizando la permeabilidad de la vía aérea, la ventilación y la circulación, instaurando en el tiempo requerido las medidas de inmovilización espinal. Al tener la estabilidad del paciente se procede a la realización de una evaluación neurológica. Si el paciente 
esta inconsciente se debe considerar la presencia de lesión medular y sospechar si presenta signos de paresia de extremidades o parálisis, pérdida de la sensibilidad, dificultad en lenguaje, hipotensión arterial, respiración diafragmática, dolor en la columna vertebral, priapismo, incontinencia urinaria y rectal. Si el paciente se encuentra activo y alerta se procede a realizar una evaluación sensitiva y motora más detallada y específica. (Bustamante, 2021).

El daño neurológico se clasifica en completo o incompleto, según el compromiso a nivel sacro. La función de esfínteres y persistencia de sensibilidad perineal se asocian a lesiones incompletas y de pronóstico favorable. Las lesiones ubicadas en niveles más altos de la médula espinal están asociadas a mayor disfunción autonómica simpática, al contrario de las lesiones que se ubican en los niveles inferiores de la columna vertebral en las que se ve comprometida la funcionalidad autonómica parasimpática generando priapismo y pérdida en la funcionalidad de la vejiga urinaria y el intestino. (Galeiras et al., 2017).

La exploración radiológica en el traumatismo raquimedular genera un diagnóstico más preciso. La radiografía simple es un método tradicional, sin embargo, la tomografía helicoidal es más sensible, más específica y es el tipo de imagen de elección. Permite visualizar fracturas que pueden pasar desapercibidas con las radiografías simples, genera una proyección completa de la columna con lo que se puede caracterizar mejor la invasión del canal y es de utilidad en el tratamiento quirúrgico (Galeiras et al., 2017).

Las complicaciones reportadas en la lesión medular conllevan a deterioro neurológico progresivo e incluso la muerte. Las complicaciones cardiovasculares se asocian a pérdida de tono simpático, hipotensión arterial y bradicardia. Las complicaciones de origen respiratorio incluyen la parálisis de los músculos respiratorios dependiendo del nivel de la lesión y edema pulmonar. El shock medular es otro tipo de complicación en la cual se produce parálisis motora flácida completa por debajo del nivel de la lesión, generando la pérdida de las respuestas a estímulos externos (Baabor et al., 2016).

\section{Conclusión}

Las características epidemiológicas en los pacientes con traumatismo raquimedular se presentan con mayor frecuencia en el género masculino y en rango de edad de 30 a 45 años. Los mecanismos de lesión más frecuentes son los accidentes automovilísticos y las caídas de altura. Los signos clínicos que se presentan con mayor 
frecuencia son la paresia de extremidades o parálisis, disfunción en la sensibilidad del tórax o en las extremidades, hipotensión e incontinencia urinaria. La región cervical es el nivel en donde las lesiones se presentan más frecuentemente según la literatura revisada. Dentro de las complicaciones reportadas más frecuentes se presenta la insuficiencia respiratoria, neumonía y shock medular, las cuales afectan el pronóstico de estos pacientes.

\section{Declaración}

El estudio se realizó con fines médicos, de acuerdo al Código de Ética y Buenas Prácticas COPE.

\section{Conflicto de intereses}

El autor declara no tener ningún conflicto de intereses.

\section{Referencias}

Arriagada, G., y Macchiavello, N. (2020). Traumatismo raquimedular. Revista Médica Clínica Las Condes, 31(5-6), 423-429. http://doi.org/10.1016/j.rmclc.2020.11.001

Baabor, M., Cruz, S., y Villalón, J. (2016). Actualización en la fisiopatología y manejo de traumatismo raquimedular. Revista Chilena de Neurocirugía, 42(2), 144-150. https://doi.org/10.36593/rev.chil.neurocir.v42i2.115

Bustamante, R. (2020). Traumatismo raquimedular. Revista Chilena de Anestesia, 50(1), 126-158. https://doi.org/10.25237/revchilanestv50n01-09

Galeiras, R., Ferreiro, M., Mourelo, M., Montoto, A. y Salvador, S. (2017). Actualización en lesión medular aguda postraumática. Revista Medicina Intensiva, 41(4), 237-247. https://doi.org/10.1016/j.medin.2016.11.002

Padilla, H., Ramos, Y., Alvis, H., Joaquin, A. y Moscote, L. (2017). Fisiopatología del trauma raquimedular. Revista Mexicana de Neurociencia, 18(5), 46-53. https:// www.medigraphic.com/pdfs/revmexneu/rmn-2017/rmn175e.pdf 
Teherán, A., Castro, O. y Frade, L. (2016). Incidencia y características del Trauma Raquimedular en un Hospital de III Nivel, Bogotá 2011-2014. Panamerican Journal of Trauma Critical Care \& Emergency Surgey, 5(3), 140-147. DOI: 10.5005/ jp-journals-10030-1157.

\section{Sobre el autor Alvaro Alejandro García Villafuerte}

Estudiante de pregrado de la carrera de Médico y Cirujano del Centro Universitario de Oriente de la Universidad de San Carlos de Guatemla. Participación en investigaciones realizadas en área de medicina interna, cirugía, ginecología, pediatría y ejercicio profesional supervisado.

Copyright (c) Alvaro Alejandro García Villafuerte

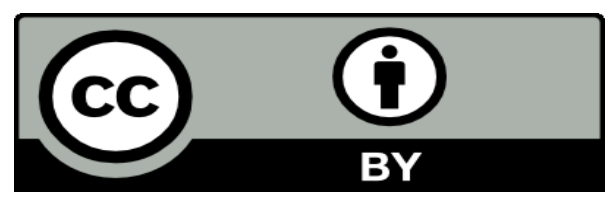

Este texto está protegido por una licencia CreativeCommons 4.0.

Usted es libre para compartir, copiar y redistribuir el material en cualquier medio o formato y adaptar el documento, remezclar, transformar y crear a partir del material para cualquier propósito, incluso comercialmente, siempre que cumpla la condición de atribución: usted debe reconocer el crédito de una obra de manera adecuada, proporcionar un enlace a la licencia, e indicar si se han realizado cambios. Puede hacerlo en cualquier forma razonable, pero no de forma tal que sugiera que tiene el apoyo del licenciante o lo recibe por el uso que hace. 